\title{
Avaliação da resistência de isolados de campo de Eimeria de frangos de corte a drogas anticoccidianas*
}

\section{Evaluation of Resistance to Anticoccidial Drugs in Field Isolates of Eimeria Oocysts from Broiler Farms}

\author{
Marcelo Vasconcelos Meireles, ${ }^{\star \star}$ Gláucia Mayumi Kavavata, ${ }^{\star \star \star}$ Sílvia Maria Almeida, ${ }^{\star \star \star}$ Maurício Hisano, ${ }^{\star \star \star \star}$ \\ Rofson Falcão Siqueira Santos ${ }^{\star \star \star \star}$
}

\begin{abstract}
Resumo
O controle da coccidiose em frangos de corte é realizado através da administração preventiva de drogas anticoccidianas na ração ou através de vacinas. No entanto, alguns surtos são observados mesmo em lotes medicados. Existem várias causas possiveis para a ocorrência de falhas na profilaxia da coccidiose, entre elas, a resistência do parasito às drogas utilizadas. Estudos epidemiológicos têm demonstrado resistência a drogas anticoccidianas em vários países, inclusive no Brasil. $O$ presente experimento teve como objetivo a determinação de resistência a drogas anticoccidianas de isolados de Eimeria de frangos de corte. Foram realizados dez experimentos, utilizando-se, em cada um, isolados de Eimeria de um complexo avícola de corte. Foi analisada resistência contra nicarbazina, robenidina, monensina, narasina, salinomicina, maduramicina, diclazuril, semduramicina e lasalocida, através da análise do ganho de peso e escore de lesões de aves infectadas, comparados aos de aves não infectadas. Os resultados observados indicaram resistência de E. tenella e E. acervulina a todas as drogas utilizadas, com exceção do diclazuril.
\end{abstract}

Palavras-chave: coccidiose, drogas anticoccidianas, resistência, frangos de corte, Eimeria.

\begin{abstract}
In broiler chicken, coccidiosis control is undertaking by using a preventive administration of anticoccidial drugs in feed or through vaccines. However, some outbreaks have been observed even in medicated flocks. There are many possible causes of failure in coccidiosis prophylaxis, such as parasite resistance to drugs. Epidemiological studies have demonstrated resistance to anticoccidial drugs in many countries, including Brazil. This experiment has undertaken to determine the pattern of anticoccidial resistance drugs of field isolates of Eimeria from broiler chickens. Drug resistence was evaluated in field Eimeria isolates from 10 poultry industries to the following drugs: nicarbazin, robenidin, monensin, narasin, salinomicin, maduramicin, diclazuril, semduramicin and lasalocid. The parameters analyzed were weight gain and lesions scores of infected birds compared to that of the uninfected ones. Results analysis demonstrated that the E. tenella and E. acervulina were resistant to all drugs tested, except for the diclazuril.
\end{abstract}

Keywords: Coccidiosis, anticoccidial drugs, resistance, broiler chickens, Eimeria

\section{Introdução}

A coccidiose em galinhas é causada por protozoários do gênero Eimeria, e se constitui, apesar dos constantes avanços concernentes à sua profilaxia, em uma das principais causas de perdas econômicas para a indústria avícola, principalmente de frangos de corte.

Estudo realizado para avaliar o impacto econômico da coccidiose no Brasil revelou que se $10 \%$ do plantel brasileiro fosse afetado por essa parasitose, haveria uma perda anual de aproximadamente 13 milhões de dólares. O mesmo autor, em levantamento realizado junto a várias empresas avícolas brasileiras, concluiu que a percentagem de lotes afetados por coccidiose clínica ou subclínica variou, entre as diferentes empresas, de um a $15 \%$. (Castro, 1994).

Atualmente, o meio mais utilizado para controle da coccidiose em frangos de corte é a administração preventiva e contínua de agentes anticoccidianos na ração. Essas drogas são utilizadas para prevenir a coccidiose em frangos de corte em todos os países que dispõem de criações em nível industrial (Reid, 1975; McDougald e Reid, 1985).

Mesmo com o uso contínuo de drogas anticoccidianas, o controle dessa enfermidade nem sempre é efetivo, o que leva à

\footnotetext{
* Suporte Financeiro: Fundação de Amparo à Pesquisa do Estado de São Paulo - FAPESP/Proc. no 98/05976-4.

** Departamento de Clínica, Cirurgia e Reprodução Animal - Curso de Medicina Veterinária - Unesp; Rua Clóvis Pestana, 793, 16050-680 Jd. Dona Amélia C. P. 341 Araçatuba - São Paulo - e-mail: marcelo@ fmva.unesp.br

*** Bolsista de Iniciação Científica - FAPESP

****Bolsista de Iniciação Científica - CNPq
} 
ocorrência de surtos mesmo em lotes medicados. Sendo assim, são descritas várias causas possíveis para ocorrência de falha profilática da coccidiose; entre elas, a resistência do parasito às drogas utilizadas, espectro de ação da droga limitado a algumas espécies, medicação interrompida ou em nível insuficiente na ração, condições climáticas e manejo deficiente, redução do consumo de ração, doenças associadas e imunossupressão (Mathis e McDougald, 1984; Peeters et al., 1994).

A resistência a drogas anticoccidianas foi reconhecida pela primeira vez por Waletzky et al. (1954), mas problemas comerciais decorrentes dessa resistência só ocorreram após a introdução do glicomide no mercado em 1956 (Reid, 1975).

A resistência a agentes químicos se desenvolve após poucos anos de uso no campo (Chapman, 1982), enquanto que com os antibióticos ionóforos o processo é mais lento (Chapman, 1976; Jeffers, 1981).

Apesar disso, o uso intensivo de antibióticos ionóforos nos últimos anos resultou em desenvolvimento de resistência também a esse grupo de anticoccidianos, o que dificulta ainda mais a escolha de um programa eficaz de combate à coccidiose, levando à limitação no uso comercial de várias drogas (Jeffers, 1974; Ryley, 1980; Reid, 1975; Chapman, 1984).

Levantamentos epidemiológicos têm demonstrado resistência a várias drogas anticoccidianas na Inglaterra (Chapman, 1982a), na antiga Tchecoslováquia (Bedrnik et al., 1985), Holanda (Voeten e Janssen, 1985), Estados Unidos (Jeffers, 1974; McDougald et al., 1986), Brasil (McDougald et al., 1987; Kawazoe e Di Fabio, 1994), Argentina (McDougald et al., 1987) e Bélgica (Peeters et al., 1994).

Esses relatos indicam resistência ou sensibilidade reduzida à maioria das drogas anticoccidianas, como robenidina, clopidol, quinolonas, halofuginona, amprólio, zoalene, nicarbazina e antibióticos ionóforos como monensina, salinomicina e lasalocida (Mathis e McDougald, 1982; Rotibi et al., 1989).

Observa-se, ainda, que a resistência às drogas tem aumentado com o passar do tempo. As cepas de campo examinadas por Jeffers (1978) foram susceptíveis à monensina, mas em 1986 somente 33\% das amostras isoladas foi susceptível a essa droga (McDougald et al., 1986).

Trabalhos mais recentes (Chapman, 1993) relatam o surgimento de resistência a praticamente todas as drogas utilizadas comercialmente.

Chapman e Hacker (1994) observaram que granjas que utilizavam extensivamente a monensina e salinomicina apresentaram cepas de Eimeria resistentes a essas drogas. Esses autores observaram ainda que, em granjas que não utilizaram lasalocida como anticoccidiano, havia a presença de cepas de Eimeria resistentes a esse agente, talvez devido a uma possivel resistência cruzada, mesmo que parcial, entre drogas ionóforas.

$A$ resistência às drogas anticoccidianas tem sido demonstrada mesmo em drogas introduzidas mais recentemente no mercado, como a maduramicina e o diclazuril (Peeters et al., 1994; Kawazoe e Di Fabio, 1994).
Várias alternativas têm sido propostas para minimizar as conseqüências do desenvolvimento de resistência aos agentes anticoccidianos, como mudar a droga utilizada logo que seja observada a resistência, alternância de drogas e utilização de combinação de drogas. Em qualquer dessas situações, para que o programa profilático seja efetivo, devem ser realizados testes de resistência em cepas de Eimeria locais, para se verificar quais agentes seriam mais efetivos contra esse parasito (Chapman, 1984).

Em relação à avaliação da resistência a agentes anticoccidianos, Ruff (1993) considera que o uso mais amplo de testes de sensibilidade que ofereçam bases mais racionais para a seleção de anticoccidianos específicos seria de grande valor para a indústria avícola, sendo particularmente úteis, segundo Rotibi et al. (1989), em periodos de alto desafio, em que a possibilidade de surtos de eimeriose é maior.

Juntamente com estudos relacionados à resistência às drogas anticoccidianas, é importante a realização de levantamentos epidemiológicos sobre os tipos de drogas e programas utilizados pela indústria avícola (Reid e McDougald, 1981), e também sobre as espécies de Eimeria predominantes em determinada região, já que algumas drogas têm uma melhor ação sobre espécies distintas desse parasito (Peeters et al., 1994), obtendo assim, informações valiosas sobre a relação do padrão de resistência com a forma e/ou continuidade de utilização de determinado produto.

No Brasil, existem somente dois trabalhos sobre resistência a agentes anticoccidianos (McDougald et al., 1987; Kawazoe e Di Fabio, 1994), o que torna difícil e muitas vezes infrutífera a escolha de um programa eficiente de combate à coccidiose em nosso país.

Devido à escassez de informações no território brasileiro sobre a coccidiose aviária, elaborou-se o presente experimento para esclarecer alguns aspectos referentes à profilaxia e epidemiologia dessa importante parasitose em plantéis de frangos de corte, como um passo inicial para estudos mais aprofundados sobre a eimeriose aviária no território nacional.

\section{Material e métodos}

As aves foram alojadas em um conjunto de gaiolas com aquecimento elétrico, permanecendo, até o início do período experimental (14 dias de vida), 30 aves por andar, após o quê, foram transferidas para gaiolas, permanecendo dez aves por unidade.

O experimento foi realizado em instalações pertencentes ao curso de Medicina Veterinária da Faculdade de Odontologia UNESP - Campus de Araçatuba. As aves foram alojadas no infectório de Ornitopatologia, o processamento das amostras foi feito no laboratório de Ornitopatologia e a ração foi manufaturada na fábrica de ração do Setor de Zootecnia.

Foram utilizados pintos de corte, machos, de linhagem comercial, vacinados no primeiro dia de vida contra a doença de Marek e provenientes de matrizes imunizadas contra as principais enfermidades que acometem essa espécie animal.

As aves foram agrupadas com base em peso para formação de parcelas homogêneas e distribuídas, aleatoriamente, em 
11 tratamentos e duas repetições, com dez aves por parcela, totalizando 220 aves por experimento. A distribuição dos pintos em lotes de aves experimentais ocorreu aos 14 dias de idade, precedendo naturalmente a inoculação de oocistos esporulados do gênero Eimeria.

Foram realizados 10 experimentos, utilizando-se, em cada um, uma mistura de amostras de fezes de um complexo avícola de corte, sendo nove do estado de São Paulo e um do estado do Paraná.

Em cada experimento, amostras de fezes foram colhidas em três a cinco galpões de frangos de corte de uma mesma empresa, com aves variando de 30 a 40 dias de idade. Dessas amostras foi formado um pool que foi acondicionado em frascos de plástico contendo solução de bicromato de potássio $2 \%$ e enviadas ao laboratório, sendo posteriormente submetidas a um processo de purificação e concentração de oocistos em solução salina saturada. Após esta etapa, procedeu-se à diluição dos oocistos em bicromato de potássio $2 \%$ e aeração forçada em temperatura de $30^{\circ} \mathrm{C}$, para que ocorresse a esporulação dos oocistos.

Não foram feitas tentativas para isolar colônias puras de Eimeria. A identificação de E. acervulina, E. maximae E. tenella foi feita através da análise do local e aparência das lesões macroscópicas e da análise morfológica e morfométrica dos oocistos e estágios endógenos.

Para determinação da dose infectante ideal utilizada no teste de resistência foi feito um pré-teste para cada experimento, no qual dois grupos de cinco aves de aproximadamente duas semanas de vida, sem medicação, foram inoculados com oocistos esporulados presentes na mistura de amostras de determinada empresa, com doses crescentes de $2 \times 10^{4}, 5 \times$ $10^{4}, 1 \times 10^{5}, 2 \times 10^{5}$ e $1 \times 10^{6}$ oocistos totais (sem identificação da espécie).

As aves foram necropsiadas seis dias após a inoculação. A dosagem que determinou escore de lesões variando de 2 a 3 , causadas por uma ou mais espécies de Eimeria, segundo a escala de Johnson e Reid (1970), sem presença de mortalidade, foi utilizada para inoculação das aves para realização do teste de resistência.

Vinte e quatro horas antes da inoculação de oocistos de Eimeria, as seguintes drogas foram incorporadas à ração em dosagens recomendadas pelos fabricantes para uso comercial: nicarbazina $(125 \mathrm{ppm})$, robenidina (33 ppm), monensina $(110 \mathrm{ppm})$, narasina $(80 \mathrm{ppm})$ salinomicina $(60$ $\mathrm{ppm})$, maduramicina (5ppm), diclazuril (1 $\mathrm{ppm})$, semduramicina (25 ppm) e lasalocida (90 ppm).

Foi utilizado um delineamento inteiramente casualizado, com 11 tratamentos e duas repetições, com dez aves por parcela, assim distribuídos (Chapman, 1998):

T1 a T9: Aves infectadas + ração com 1 agente anticoccidiano;

T10: Aves infectadas e não medicadas;

T11: Aves não infectadas e não medicadas.

No sexto dia após a inoculação, as aves foram necropsiadas e, a seguir, foi determinado o escore de lesões segundo a técnica de Johnson e Reid (1970).

A sensibilidade de um determinado isolado foi estabelecida conforme Rotibi et al. (1989), através da análise de dois parâmetros:
1- Percentagem de ganho de peso do grupo de aves infectadas comparado ao do grupo controle não infectado, onde:

- grupo controle não infectado e não medicado $=100 \%$

2 - Percentagem de redução do escore de lesões dos grupos de aves infectadas comparado ao do grupo controle não infectado, onde:

- grupo controle não infectado e não medicado: percentagem de redução de lesão $=100 \%$.

- grupo controle infectado e não medicado: percentagem de redução de lesão $=0 \%$.

A partir da soma desses dois parâmetros foi estabelecido um "índice de eficiência anticoccidiana". Um isolado foi considerado resistente a determinada droga quando a média do grupo infectado foi menor que $50 \%$ da do grupo controle não infectado.

Para determinação do ganho de peso, as aves foram pesadas no dia da inoculação e sete dias após, antes da realização da necropsia.

\section{Resultados e discussão}

Pela análise do local parasitado, aspectos das lesões macroscópicas e análise morfológica e morfométrica dos oocistos, pôde ser feita a identificação das três espécies mais comuns de frangos de corte, E. acervulina, E. maxima e $E$. tenella.

A existência de várias espécies em proporções diferentes no inóculo não permitiu que o cálculo do índice de eficiência anticoccidiana fosse feito para todas as espécies, pois a $E$. acervulina apresentou número suficiente de oocistos para a realização do teste de resistência no primeiro, segundo, terceiro e sexto experimentos. Para E. tenella, foi possível o cálculo deste índice no segundo, quarto, quinto, sexto, sétimo e oitavo, nono e décimo experimentos.

Foi observado ainda que, apesar da $E$. maxima estar presente nas amostras de todos os experimentos, esta espécie, mesmo com um grande número de oocistos presente nos inóculos, não promoveu lesões características como as descritas por Johnson e Reid (1970), impossibilitando, assim, o cálculo do índice de eficiência anticoccidiana. Esse cálculo, segundo os resultados encontrados neste experimento, torna-se viável somente com inóculos puros contendo esta espécie e, mesmo assim, a classificação em escores se torna difícil pela subjetividade na interpretação de suas lesões macroscópicas.

$O$ índice de eficiência anticoccidiana adotado neste experimento para determinação da presença de resistência de isolados de campo de Eimeria leva em consideração a redução o ganho de peso e o escore de lesão das aves infectadas comparados aos de aves não infectadas, em infecções mistas, segundo Rotibi et al. (1989). Não existe na literatura padronização dos parâmetros utilizados para determinar a ocorrência de resistência a drogas anticoccidianas. A determinação do "ponto de corte" a partir do qual um isolado é considerado resistente, ou segundo alguns autores, parcialmente resistente, é realizada de maneira arbitrária pelo pesquisador, e sem dúvida influencia no número de isolados considerados como resistentes a determinada droga (Chapman, 1998). 
Nas Tabelas 1, 2 e 3; observa-se que, após o cálculo do índice de eficiência anticoccidiana, foram encontradas amostras de $E$. acervulina e $E$. tenella resistentes a quase todas as drogas utilizadas, com exceção do diclazuril.

Os isolados de $E$. acervulina apresentaram resistência variando de $0 \%$ para o diclazuril até $50 \%$ para semduramicina, maduramicina, robenidina e monensina. Dos isolados de $E$. tenella, $0 \%$ foram resistentes ao diclazuril, $12,5 \%$ resistentes à robenidina e até $75 \%$ das amostras resistentes à monensina.

Os resultados observados em outros experimentos (Mathis e McDougald, 1982; Rotibi et al., 1989; Bedrnik et al., 1985; Voeten e Janssen, 1985; McDougald et al., 1986; McDougald et al., 1987; Peeters et al., 1994) estão de acordo com os do presente experimento e demonstram presença de resistência das amostras de Eimeria às drogas ionóforas. Provavelmente, isso se deve ao fato dessa classe de drogas ser utilizada em larga escala, e há muito tempo, pela indústria avícola de frangos de corte.

Embora tenha sido encontrada resistência a drogas ionóforas, principalmente relacionada a E. tenella, nos lotes onde as amostras foram colhidas não foram observados surtos de coccidiose clínica. Isso provavelmente se deve ao fato de que essas drogas permitem, durante sua administração contínua no campo, o desenvolvimento gradual de imunidade por parte das aves. Desse modo, apesar de, em muitos casos, não se mostrarem totalmente eficientes para impedir a infecção, o desenvolvimento da imunidade apresenta efeito sinérgico, impedindo o desenvolvimento de doença clínica e mortalidade.

De qualquer maneira, deve-se levar em conta que, nos casos em que houver alto desafio por Eimeria sp, a utilização de uma droga que já apresenta perda de eficácia pode levar ao desenvolvimento de doença clínica com presença de mortalidade, ou mesmo, a perdas econômicas imperceptíveis decorrentes de coccidiose subclínica (Ruff, 1993).

Já em relação aos anticoccidianos químicos, observa-se que o diclazuril, uma das drogas mais recentes do mercado, apre- sentou excelente eficácia contra todos os isolados de ambas as espécies. Apesar disso, essa droga deve ser utilizada somente em ocasiões em que outros agentes anticoccidianos mostraram-se ineficientes, pois já foi demonstrado, inclusive no Brasil, que existem cepas que já desenvolveram resistência contra essa droga (Kawazoe, 1994).

A nicarbazina continua sendo uma das drogas mais utilizadas na primeira fase de crescimento das aves, principalmente durante os meses de inverno. Talvez essa periodicidade de utilização seja responsável pelo fato de que tenham sido encontrados somente dois isolados resistentes a essa droga.

Quanto à robenidina, foram encontrados dois isolados resistentes, um de E. acervulina e um de E. tenella. Apesar de atualmente ser utilizada esporadicamente devido ao rápido surgimento de resistência (Chapman, 1982), essa droga deve ser administrada com cautela, por períodos curtos, e somente após a realização de testes de resistência a drogas anticoccidianas.

Os programas de profilaxia anticoccidiana utilizados hoje no Brasil são baseados principalmente na adição à ração de nicarbazina ou associação de nicarbazina a um ionóforo, na fase inicial, e de um ionóforo na fase de crescimento. A robenidina e o diclazuril são utilizados esporadicamente em programas duais ou durante toda a fase de criação.

Tabela 1: Número de isolados de campo resistentes a drogas anticoccidianas

\begin{tabular}{lcc}
\hline Compostos & $\begin{array}{c}\text { Número de isolados resistentes/número de } \\
\text { isolados testados }\end{array}$ \\
\hline Narasina & E. acervulina & $\begin{array}{c}\text { E. tenella } \\
\text { Lasalocida }\end{array}$ \\
Semduramicina & $1 / 4$ & $5 / 8$ \\
Diclazuril & $1 / 4$ & $5 / 8$ \\
Maduramicina & $2 / 4$ & $2 / 8$ \\
Salinomicina & $0 / 4$ & $0 / 8$ \\
Robenidina & $2 / 4$ & $5 / 8$ \\
Monensina & $1 / 4$ & $3 / 8$ \\
Nicarbazina & $1 / 4$ & $1 / 8$ \\
\hline
\end{tabular}

Tabela 2: Avaliação de resistência a agentes anticoccidianos de amostras de campo. Experimentos 1 a 5

\begin{tabular}{|c|c|c|c|c|c|c|}
\hline \multirow{3}{*}{ Tratamento } & \multicolumn{6}{|c|}{ Índice de eficiência anticoccidiana ${ }^{\star}$} \\
\hline & \multirow{2}{*}{$\frac{1^{\circ}}{\text { E. acervulina }}$} & \multicolumn{2}{|c|}{$2^{\circ}$} & \multirow{2}{*}{$\frac{3^{\circ}}{\text { E. acervulina }}$} & \multirow{2}{*}{$\frac{4^{\circ}}{\text { E. tenella }}$} & \multirow{2}{*}{$\frac{5^{\circ}}{\text { E. tenella }}$} \\
\hline & & E. acenulina & E. tenella & & & \\
\hline Não Infectado/ Não Medicado & 200 & 200 & 200 & 200 & 200 & 200 \\
\hline Infectado/ Não Medicado & 64 & 61 & 61 & 65 & 52 & 44 \\
\hline Narasina & 136 & 103 & 83 & 111 & 84 & 92 \\
\hline Lasalocida & 128 & 118 & 98 & 128 & 95 & 81 \\
\hline Semduramicina & 131 & 138 & 103 & 99 & 119 & 71 \\
\hline Diclazuril & 113 & 183 & 183 & 130 & 204 & 120 \\
\hline Maduramicina & 115 & 99 & 94 & 199 & 81 & 56 \\
\hline Salinomicina & 119 & 116 & 116 & 128 & 83 & 60 \\
\hline Robenidina & 116 & 200 & 160 & 89 & 126 & 82 \\
\hline Monensina & 103 & 95 & 80 & 122 & 110 & 53 \\
\hline Nicarbazina & 90 & 113 & 162 & 128 & 145 & 131 \\
\hline
\end{tabular}

* Índice de eficiência anticoccidiana menor que 100 indica resistência à droga utilizada. 
Tabela 3: Avaliação de resistência a agentes anticoccidianos de amostras de campo. Experimentos 6 a 10

\begin{tabular}{|c|c|c|c|c|c|c|}
\hline \multirow{3}{*}{ Tratamento } & \multicolumn{6}{|c|}{ Índice de eficiência anticoccidiana* } \\
\hline & \multicolumn{2}{|c|}{$6^{\circ}$} & \multirow{2}{*}{$\frac{7^{\circ}}{\text { E. tenella }}$} & \multirow{2}{*}{$\frac{8^{\circ}}{\text { E. tenella }}$} & \multirow{2}{*}{$\frac{9^{\circ}}{\text { E. tenella }}$} & \multirow{2}{*}{$\frac{10^{\circ}}{\text { E. tenella }}$} \\
\hline & E. acervulina & E. tenella & & & & \\
\hline $\begin{array}{l}\text { Não Infectado/ Não } \\
\text { Medicado }\end{array}$ & 200 & 200 & 200 & 200 & 200 & 200 \\
\hline $\begin{array}{l}\text { Infectado/ Não } \\
\text { Medicado }\end{array}$ & 45 & 45 & 55 & 55 & 65 & 56 \\
\hline Narasina & 60 & 57 & 108 & 110 & 84 & 112 \\
\hline Lasalocida & 80 & 79 & 140 & 120 & 105 & 90 \\
\hline Semduramicina & 55 & 84 & 120 & 146 & 105 & 115 \\
\hline Diclazuril & 105 & 151 & 165 & 155 & 175 & 156 \\
\hline Maduramicina & 94 & 87 & 90 & 133 & 130 & 142 \\
\hline Salinomicina & 51. & 106 & 90 & 141 & 106 & 115 \\
\hline Robenidina & 110 & 158 & 175 & 105 & 146 & 125 \\
\hline Monensina & 58 & 84 & 96 & 105 & 85 & 95 \\
\hline Nicarbazina & 102 & 91 & 170 & 154 & 125 & 146 \\
\hline
\end{tabular}

* Indice de eficiência anticoccidiana menor que 100 indica resistência à droga utilizada.

Os resultados obtidos nos permitem concluir que os programas anticoccidianos utilizados atualmente pela indústria avícola, tendo como base principalmente os ionóforos e a nicarbazina ainda são eficientes, em muitos casos, para a prevenção do surgimento de coccidiose clínica, mesmo que as amostras isoladas se apresentem resistentes, em testes laboratoriais, às drogas utilizadas. No entanto, precauções extras devem ser adotadas para um monitoramento rigoroso para a deteç̧ão de infecções subclínicas, principalmente quando são utilizados agentes ionóforos durante os meses de alto desafio.

A utilização de agentes químicos, principalmente o diclazuril e a robenidina, apresenta-se como alternativa para ocasiōes

\section{Referências}

BEDRNIK, P., JURKOVIC, P., SEVCIK, B., FIRMANOVA, A., KUCERA, J. Sensitivity of Czechoslsovak field isolates of Eimeria tenella to monensin, lasalocid and arprinocid. Arch. Gefluegelkd. v. 49, p. 1-6, 1985.

CASTRO, A.G.M. Situação atual da coccidiose no Brasil e importância econômica. SIMPÓSIOINTERNACIONAL SOBRECOCCICIOSE, 1., 1994, Santos. Anais..., 1994. p. 45-54.

CHAPMAN, H.D. Eimeria tenella in chickens: studies on resistance to the anticoccidial drugs monensin and lasalocid. Vet. Parasitol., v. 2, p. 187-196, 1976.

Anticoccidial drug resistance. In: LONG, P.L. (Ed.). The Biology of Coccidia. Baltimore: University Park Press, 1982, p. 429-452.

The sensitivity of field isolates of Eimeria acervulina type to monensin. Vet. Parasitol, v. 9, p. 179-183, 1982a. em que outros agentes anticoccidianos forem ineficientes.

\section{Conclusões}

- Existem cepas de E. acervulina e E. tenella nas granjas de frangos de corte dos estados de São Paulo e Paraná resistentes a drogas anticoccidianas, de acordo com os critérios utilizados neste experimento.

- A resistência ocorre predominantemente contra agentes ionóforos.

- A presença de resistência não está necessariamente associada à coccidiose clínica no campo.

CHAPMAN, H.D. Drug resistance in avian coccidia (a review). Vet. Parasitol., v. 15, p. 11-27, 1984.

Evaluation of the efficacy of anticoccidial drugs against Eimeria species in the fowl. Int. J. Parasitol., v. 28, p. 1.141-1.144, 1998.

9, p. 150-161, 1993

Resistance to anticoccidial drugs in fowl. Parasitol. Today, v.

CHAPMAN, H.D., HACKER, A.B. Sensitivity of field isolates of Eimeria from two broiler complexes to anticoccidial drugs in the chicken. $P$. Sci., v. 73, p. 1.404-1.408, 1994.

JEFFERS, T.K. Eimeria Tenella incidence, distribution, and anticoccidial drug resistence of isolates in major broiler-producing areas. Avian Dis., v. 18, p. $74-84,1974$.

Eimeria tenella: sensitivity of recent isolates field isolates to monensin. Avian Dis., v. 22, p. 157-161, 1978.

Sensitivity of field strains of coccidia to monensin: the first 10 years of drug exposure. P. Sci., v. 60, p. 1675, 1981. 
JOHNSON, J., REID, W.M. Anticoccidial drugs: lesion scoring techniques in battery and floor-pen experiments. Exp. Parasitol., v. 28, p. 30-36, 1970. JORGENSEN, W.K., STEWART, N.P., JESTON, P.J., MOLOY J.B., BLIGHT G.W., ALGLIESHR.J. Isolation and pathogenicity of Australian strains of Eimeria praecox and Eimeria mitis. Aust. Vet. J., v. 75, p. 592-595, 1997.

KAWAZOE, U., DI FABIO, J. Resistance to diclazuril in field isolates of Eimeria species obtained from commercial broiler flocks in Brazil. Avian Pathol., v. 23, p. 305-311, 1994.

McDOUGALD, L.R., DA SILVA, J.M.L., SOLIS, J., BRAGA, M. A survey of sensitivity to anticoccidial drugs in 60 isolates of coccidia from broiler chickens in Brazil and Argentina. Avian Dis., v. 31, p. 287-292, 1987.

McDOUGALD, L.R., FULLER, L., MATTIELLO, R. A survey of coccidia on 43 poultry farms in Argentina. Avian Dis., v. 41, p. 923-929, 1997.

MCDOUGALD, L.R., FULLER, L., SOLIS, J. Drug-sensitivity of 99 isolates of coccidia from broiler farms. Avian Dis., v. 30, p. 690-694, 1986.

McDOUGALD, L.R; REID, W.M. Coccidiosis control, past and future. Broiler Industry, v. 8, p. 34-41, 1985.

MATHIS, G.F., McDOUGALD, L.R. Drug responsiveness of field isolates of chickens coccidia. P. Sci., v. 61, p. 38-45, 1982.

MATHIS, G.F., McDOUGALD, L.R. Effectiveness of therapeutic anticoccidial drugs against recently isolated coccidia. P. Sci., v. 63, p. 1.149-1.153, 1984.
PEETERS, J.E., DERIJCKE, J., VERLINDEN, M., WYFFELS, R. Sensitivity of avian Eimeria spp. to seven chemical and five ionophore anticoccidials in five Belgian integrated broiler operations. Avian Dis., v. 38, p. 483-493, 1994.

REID, W.M. Progress in the control of coccidiosis with anticoccidials and planned immunization. Am. J. Vet. Res., v. 36, p. 593-595, 1975.

REID, W.M., McDOUGALD, L.R. New drugs to control coccidiosis. Feedstuffs, v. 53, p. 27-28, 1981.

ROTIBI, A., McDOUGALD, L.R., SOLIS, J. Response to 21 Canadian field isolates of chicken coccidia to commercial anticoccidial drugs. Avian Dis., v. 33, p. 365-367, 1989.

RUFF, M.D. Valor de la prueba de sensibilidad en coccidiosis aviar. Avic. Prof., v. 10, p. 109-116, 1993.

RUFF, M.D., EDGAR, S.A. Reduced intestinal absorption in broilers during Eimeria mitis infection. Am. J. Vet. Res.. v. 43, p. 507-509, 1982.

RYLEY, J.F. Drug resistance in coccidia. Adv. Vet. Sci. Comp. Med., v. 24, p. 99-120, 1980.

VOETEN, A.C., JANSSEN, B.A.P.M. A study of the effects of replacing monensin ionophore with lasalocid ionophore in the field control of coccidiosis in broiler. Vet. Q., v. 7, p. 66-69, 1985.

WALETZKY, E., NEAL, R., HABLE, I. A field strain of Eimeria Tenella resistant to sulfonamides (abstr). J. Parasitol., v. 40, p. 24, 1954. 